\title{
Severe Alcoholic Hepatitis: Atypical Presentation with Markedly Elevated Alkaline Phosphatase
}

\author{
Page Axley ${ }^{1}$, Kirk Russ ${ }^{2}$ and Ashwani K. Singal*2 \\ ${ }^{1}$ Tinsley Harrison Internal Medicine Residency Program, University of Alabama at Birmingham, Birmingham, AL, USA; ${ }^{2}$ Division \\ of Gastroenterology and Hepatology, University of Alabama at Birmingham, Birmingham, AL, USA
}

\begin{abstract}
Alcoholic hepatitis $(\mathrm{AH})$ is an acute inflammatory liver disease with poor prognosis. Infections in $\mathrm{AH}$ are difficult to detect and contribute to short-term mortality. Intrahepatic cholestasis and elevated alkaline phosphatase levels are also associated with worse outcomes. This report describes an uncommon presentation of severe $\mathrm{AH}$.

Citation of this article: Axley P, Russ K, Singal AK. Severe alcoholic hepatitis: atypical presentation with markedly elevated alkaline phosphatase. J Clin Transl Hepatol 2017; 5(4):414-415. doi: 10.14218/JCTH.2017.00044.
\end{abstract}

\section{Case report}

A 53-year-old woman was transferred from another hospital with progressive jaundice and lower extremity edema that had lasted for 6 weeks. She was previously treated for 2 weeks with prednisolone for suspected alcoholic hepatitis $(\mathrm{AH})$. She had a long history of heavy alcohol use and her last drink was 4 weeks prior to admission. On arrival, her temperature was $96.5^{\circ} \mathrm{F}$, pulse was $97 / \mathrm{min}$, respiratory rate was $16 / \mathrm{min}$, and blood pressure was $118 / 82$. Physical examination was significant for icteric skin and sclera, non-tender obese abdomen with hepatomegaly, and lower extremity pitting edema. Laboratory profile showed serum bilirubin of $22.2 \mathrm{mg} / \mathrm{dL}$, direct bilirubin of $14.8 \mathrm{mg} / \mathrm{dL}$, alanine aminotransferase (ALT) of $85 \mathrm{U} / \mathrm{L}$, aspartate aminotransferase (AST) of $242 \mathrm{U} / \mathrm{L}$, alkaline phosphatase ALP $805 \mathrm{U} / \mathrm{L}$, gamma-glutamyl transferase (GGT) of $2582 \mathrm{U} / \mathrm{L}$, white blood cell count (WBC) of $23.8 \mu \mathrm{L}$, and ammonia of $89 \mathrm{mg} / \mathrm{dL}$. Liver enzymes were elevated from previous lab work done 2 weeks prior (AST of 196, ALT of 34, ALP of 338).

The patient was not taking any medications known to cause cholestasis. Her medications on admission included furosemide, spironolactone, prednisolone, and lactulose. Her Lille score was 0.9 , suggesting poor response to steroids, and prednisolone was discontinued. Infectious studies were negative, including urine cultures, blood cultures, chest

Keywords: Alcoholic hepatitis; Alcoholic liver disease; Alkaline phosphatase; Cholestatic liver disease.

Abbreviations: $\mathrm{AH}$, alcoholic hepatitis; ALP, alkaline phosphatase; ALT, alanine aminotransferase; AST, aspartate aminotransferase; ESBL, extended-spectrum beta-lactamase; GGT, gamma-glutamyl transferase; ULN, upper limit of normal; WBC, white blood cell.

Received: 21 July 2017; Revised: 5 August 2017; Accepted: 14 August 2017

*Correspondence to: Ashwani K. Singal, Division of Gastroenterology and Hepatology, University of Alabama Birmingham, AL 35294-0012, USA. Tel: +1-205975-9698, Fax: +1-205-975-0961, E-mail: ashwanisingal.com@gmail.com radiograph, and ascitic fluid analysis. Acute viral hepatitis ( $A, B$, and $C$ ) was ruled out by respective serological testing for each. Anti-nuclear antibody, anti-mitochondrial antibody and anti-smooth muscle antibody tests were negative. Acetaminophen and salicylate levels were undetectable.

Abdominal ultrasound showed an enlarged $(24.5 \mathrm{~cm})$ steatotic liver, cholelithiasis, and a 4-mm common bile duct. Magnetic resonance cholangiopancreatography confirmed liver enlargement without intra or extra-hepatic biliary dilation. Given the presence of cholelithiasis and jaundice, endoscopic ultrasound was performed and ruled out choledocholithiasis or extrahepatic bile duct obstruction. Transjugular liver biopsy performed 1 week after presentation showed changes consistent with severe $\mathrm{AH}$, including neutrophilic lobular infiltration, Mallory hyaline, ballooning degeneration of hepatocytes, and cholestasis of ductules and canaliculi. Trichrome stain confirmed presence of cirrhosis (Fig. 1). The patient's Maddrey's discriminant function of 35 and model for end-stage liver disease score of 22 were consistent with severe $\mathrm{AH}$.

The patient was discharged to home with outpatient follow-up scheduled at 4 days. Two days after discharge, the patient was readmitted with altered mental status. She had severe metabolic acidosis (lactic acid $18 \mathrm{mg} / \mathrm{dL}$ ), WBC of $22.2 \mu \mathrm{L}$, and ammonia level of $686 \mathrm{mg} / \mathrm{dL}$. Serum bilirubin was $10.2 \mathrm{mg} / \mathrm{dL}$, which represented an improvement from 15.6 at time of discharge. Urine culture was positive for extended-spectrum beta-lactamase (ESBL)-producing $E$. coli and blood cultures for streptococcus viridians. Unfortunately, despite aggressive resuscitative efforts, she passed away within 24 hours of re-admission.

\section{Discussion}

This case represents an atypical presentation of $\mathrm{AH}$ with marked elevation of ALP. AH is clinically diagnosed by the presence of jaundice, serum bilirubin of $>3 \mathrm{mg} / \mathrm{dL}$, AST to ALT ratio of $>1.5$ with elevated levels but not exceeding $400 \mathrm{IU} / \mathrm{L}$, heavy alcohol use (typically for $>5$ years) until at least 6 weeks prior to presentation, and exclusion of other causes of liver disease. ${ }^{1}$ ALP is elevated in $\mathrm{AH}$ patients, with levels usually within 2-3 times the upper limit of normal (ULN), even among non-survivors. ${ }^{2}$ ALP and GGT levels in our patient were $805 \mathrm{U} / \mathrm{L}$ (over 7 times ULN of 117 at our center) and 2582 IU (over 40 times ULN of 65 at our center), respectively. Mild to moderate elevation of ALP in AH patients has been well documented in the literature, but very high levels of ALP are rarely reported. In 1978, Perrillo and his colleagues ${ }^{3}$ published a case series of 20 alcoholic patients who presented 

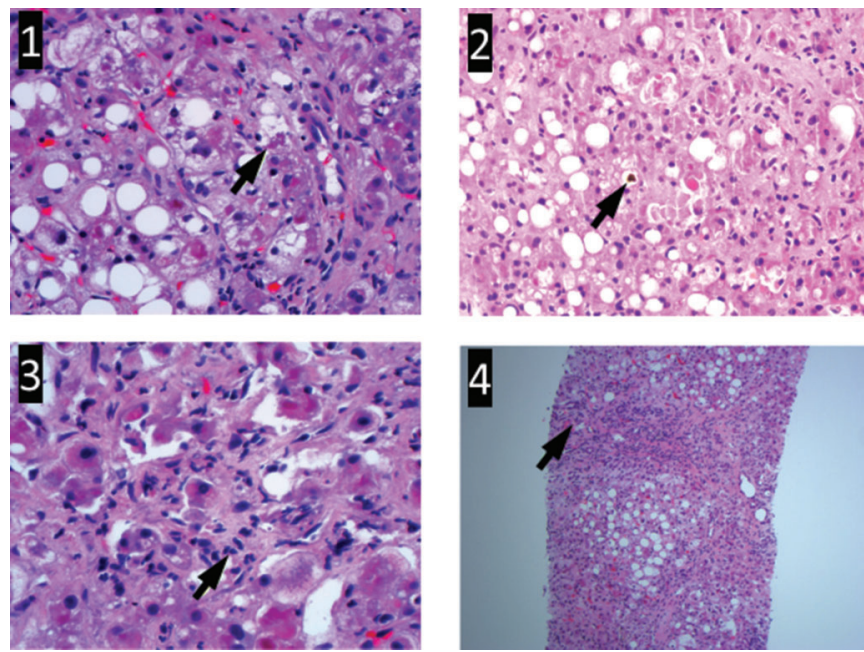

Fig. 1. Liver biopsy findings of severe alcoholic hepatitis in our patient Macrovesicular steatosis with Mallory hyaline (panel 1, arrow), intracanalicular and ductular cholestasis (panel 2, arrow), and neutrophilic infiltration of lobules and hepatocytes (panel 3, arrow) are shown. Also seen are changes of advanced bridging fibrosis to evolving cirrhosis (panel 4, arrow).

with ALP elevations of approximately 4 times the ULN (mean: 582 and SD: 37 IU/L). Given this atypical presentation of marked elevation of ALP and GGT, diligent work-up was obtained to exclude biliary obstruction, and trans-jugular liver biopsy was performed to confirm the diagnosis of $\mathrm{AH}$.

The patient's presentation was most likely secondary to infection in the setting of severe $\mathrm{AH}$. While the initial infectious workup was negative, cultures obtained on readmission showed Gram-positive bacteremia and an ESBLproducing urinary tract infection. The principal mechanisms of cholestasis caused by infection include disruption of bile flow and impairment in bilirubin metabolism. ${ }^{4}$ Infections are common in $\mathrm{AH}$ patients with prevalence of $12-26 \%$, and have negative impact on short-term survival, especially among non-responders to corticosteroids, as in our patient. ${ }^{5}$ This raises important issues regarding the protocol for infection surveillance in $\mathrm{AH}$ patients and the clinically unmet need of biomarkers for early diagnosis of infections in $\mathrm{AH}$.

Our patient had features of systemic inflammatory response syndrome (SIRS). However, SIRS is present in about $60 \%$ of $\mathrm{AH}$ patients but only $20-30 \%$ of patients are diagnosed with infection. ${ }^{6}$ Potential biomarkers are procalcitonin, lipopolysaccharide, and bacterial DNA and need to be tested in well-designed clinical trials before recommending their routine use in clinical practice. ALP level and cholestasis on liver biopsy are associated with poor patient outcome. ${ }^{5}$ In a study on $116 \mathrm{AH}$ patients, ALP > 1.5 ULN was independently associated with poor 90-day survival, with mean ALP of 169 IU/L in survivors compared to $236 \mathrm{IU} / \mathrm{L}$ in non-survivors. ${ }^{7}$ However, whether the presence of cholestasis and elevated ALP indicate infection risk remains a testable hypothesis.

\section{Conclusions}

In summary, we describe an atypical presentation of markedly elevated ALP in a patient diagnosed with severe $\mathrm{AH}$. Clinicians should be aware of this potential presentation of this common liver disease, remain vigilant for infections in these patients, and utilize liver biopsy early when diagnosis remains uncertain.

\section{Conflict of interest}

The authors have no conflict of interests related to this publication.

\section{Author contributions}

Review of literature and drafting of manuscript (PA), critical revision of the manuscript (KR, AKS).

\section{References}

[1] Crabb DW, Bataller R, Chalasani NP, Kamath PS, Lucey M, Mathurin P, et al. Standard definitions and common data elements for clinical trials in patients with alcoholic hepatitis: recommendation from the NIAAA alcoholic hepatitis consortia. Gastroenterology 2016;150:785-790. doi: 10.1053/j.gastro. 2016.02.042.

[2] Thursz MR, Richardson P, Allison M, Austin A, Bowers M, Day CP, et al. Prednisolone or pentoxifylline for alcoholic hepatitis. N Engl J Med 2015;372: 1619-1628. doi: 10.1056/NEJMoa1412278.

[3] Perrillo RP, Griffin R, DeSchryver-Kecskemeti K, Lander JJ, Zuckerman GR. Alcoholic liver disease presenting with marked elevation of serum alkaline phosphatase. A combined clinical and pathological study. Am J Dig Dis 1978;23:1061-1066. doi: 10.1007/BF01072879.

[4] Chand N, Sanyal AJ. Sepsis-induced cholestasis. Hepatology 2007;45: 230-241. doi: 10.1002/hep.21480.

[5] Louvet A, Wartel F, Castel H, Dharancy S, Hollebecque A, Canva-Delcambre V, et al. Infection in patients with severe alcoholic hepatitis treated with steroids: early response to therapy is the key factor. Gastroenterology 2009;137: 541-548. doi: 10.1053/j.gastro.2009.04.062.

[6] Michelena J, Altamirano J, Abraldes JG, Affò S, Morales-Ibanez O, Sancho-Bru $P$, et al. Systemic inflammatory response and serum lipopolysaccharide levels predict multiple organ failure and death in alcoholic hepatitis. Hepatology 2015;62:762-772. doi: 10.1002/hep.27779.

[7] Kasztelan-Szczerbinska B, Slomka M, Celinski K, Szczerbinski M. Alkaline phosphatase: the next independent predictor of the poor 90-day outcome in alcoholic hepatitis. Biomed Res Int 2013;2013:614081. doi: 10.1155/ $2013 / 614081$. 